\title{
UTILIZACIÓN DE MICROALGAS COMO ALTERNATIVA PARA LA REMOCIÓN DE METALES PESADOS
}

\author{
USE OF MICROALGAE AS AN ALTERNATIVE IN HEAVY METAL REMOVAL
}

Deimer Vitola Romero • deimervitolaromero@gmail.com

Ms.C en Ciencias Ambientales, Universidad de Sucre, Sincelejo, Colombia.

\begin{abstract}
Alexander Pérez Cordero • alexander.perez@unisucre.edu.co PhD. Microbiología Agrícola, Universidad de Sucre, Sincelejo, Colombia.
\end{abstract}

Donicer E. Montes Vergara.donicer.montes@unisucre.edu.co PhD. Genética y Mejoramiento Animal. Universidad de Sucre, Sincelejo, Colombia.

Citación: Vitola, D. Pérez, A., Montes, D. (2022). Utilización de microalgas como alternativa para la remoción de metales pesados. Revista de Investigación Agraria y Ambiental, 13(1), 195 - 203.

DOI: https://doi.org/10.22490/21456453.4568

\section{RESUMEN}

Contextualización: la contaminación directa de cuerpos de agua, por vertimientos de agua residual contaminada con metales pesados, es un problema importante que se ha agudizado en las últimas décadas, debido a que los metales pesados pueden alterar el correcto funcionamiento de los ecosistemas.

Vacío de investigación: se hace necesario y urgente la búsqueda de alternativas de remoción de contaminantes metálicos que sean de bajo costo y amigables con el ambiente. En ese sentido, la biosorción es una alternativa que cumple con estas características y su modo de uso es simple.

Propósito del estudio: se planteó como objetivo determinar la capacidad de biosorción de los metales pesados $\mathrm{Hg}, \mathrm{Cd}$ y $\mathrm{Pb}$ por las microalgas Chlorella vulgaris y Scenedesmus obliquus, inmovilizadas en fibra de estropajo (Luffa cylindrica), así como la desorción de estos como estrategia de recuperación.

Metodología: Para la determinación del $\mathrm{Cd}$ y $\mathrm{Pb}$ se utilizó un espectrofotómetro de absorción atómica de llama aire-acetileno y para el $\mathrm{Hg}$, uno de absorción atómica con vapor frío. El análisis de los metales pesados removidos se realizó en el sobrenadante y la capacidad de desorción se determinó en el sobrenadante resultante de la biomasa microalgal, la cual se trató con solución acuosa de ácido y centrifugada. Se realizó un análisis de varianza mediante un diseño completamente al azar. Las diferencias estadísticas significativas ( $p<0,05)$, se determinaron mediante la prueba de Tukey.

Resultados y conclusiones: los resultados indican diferencias significativas en la biosorción de metales pesados. Chlorella vulgaris tuvo la mayor biosorción con $94,77 \pm 1,63 \%$ para $\mathrm{Cd}, 92,45 \pm 3,95$ $\%$ de $\mathrm{Pb}$ y $81,78 \pm 1,36 \%$ de $\mathrm{Hg}$; mientras que Scenedesmus obliquus removió el 90,08 $\pm 2,69 \%$ de $\mathrm{Cd}, 86,17 \pm 1,78 \%$ de $\mathrm{Pb}$ y $80,2 \pm 5,49 \%$ de $\mathrm{Hg}$. Y con respecto a la desorción, Chlorella vulgaris presentó los promedios más altos con 97,29 $\pm 1,93 \%$ de $\mathrm{Hg}, 96,86 \pm 2,14 \%$ de Cd y $95,48 \pm 1,19 \%$ de $\mathrm{Pb}$; mientras que Scenedesmus obliquus mostró una desorción de $96,74 \pm 2,14 \%$ de $\mathrm{Hg}, 95,15 \pm 2,90 \%$ de $\mathrm{Cd}$ y $93,82 \pm 2,68 \%$ de $\mathrm{Pb}$. Estos resultados demuestran que la aplicación de biomasa microalgal inmovilizada para la biosorción de metales pesados es una alternativa de biorremediación.

Palabras claves: contaminación; Chlorella vulgaris; Scenedesmus; metales pesados; biosorción; desorción 


\section{ABSTRACT}

Contextualization: the direct contamination of bodies of water, because of dumping of wastewater contaminated with heavy metals, is a major problem that has become more acute in recent decades, because heavy metals can disrupt the proper functioning of ecosystems.

Knowledge gap: it is necessary and urgent to search for alternatives for removal of metallic contaminants, which must be low cost, and harmless for the environment. In that sense, biosorción is an alternative that meets these requirements, and its mode of use is simple.

Purpose: the objective was to determine the biosorption capacity of the heavy metals $\mathrm{Hg}, \mathrm{Cd}$ and $\mathrm{Pb}$ by the microalgae Chlorella vulgaris and Scenedesmus obliquus, immobilized in scourer fiber (Luffa cylindrica), as well as their desorption as a recovery strategy.

Methodology: the determination of heavy metals was performed with an air-acetylene flame atomic absorption spectrophotometer for the analysis of cadmium (Cd) and lead $(\mathrm{Pb})$, and a cold vapor atomic absorption spectrophotometer for mercury $(\mathrm{Hg})$. The analysis of the removed heavy metals was performed on the supernatant, and the desorption capacity was determined on the supernatant resulting from the microalgal biomass treated with aqueous acid solution and centrifuged. An analysis of variance was performed using a completely random design. The significant statistical differences $(p<$ $0,05)$ were determined by the Tukey test.

Results and conclusions: the results indicate significant differences in the removal of heavy metals, showing Chlorella vulgaris the highest biosorption of heavy metals with $94,77 \pm 1,63 \%$ for Cd; $92,45 \pm 3,95$ $\%$ for $\mathrm{Pb}$ and $81,78 \pm 1,36 \%$ for $\mathrm{Hg}$; while Scenedesmus obliquus removed $90,08 \pm$ $2,69 \%$ of $\mathrm{Cd} ; 86,17 \pm 1,78 \% \mathrm{~Pb}$ and $80,2 \pm$ $5,49 \% \mathrm{Hg}$. And with respect to desorption, Chlorella vulgaris presented the highest averages with $97,29 \pm 1,93 \%$ of $\mathrm{Hg}, 96,86 \pm$ $2.14 \%$ of $\mathrm{Cd}$ and $95.48 \pm 1.19 \%$ of $\mathrm{Pb}$, while Scenedesmus obliquus showed a desorption of $96,74 \pm 2,14 \%$ of $\mathrm{Hg} ; 95,15 \pm 2,90 \%$ of Cd and $93,82 \pm 2,68 \%$ of $\mathrm{Pb}$. This results prove that the application of immobilized microalgal biomass for the removal of heavy metals is an excellent bioremediation alternative.

Keywords: contamination; Chlorella vulgaris; Scenedesmus; heavy metal; biosorption; desorption 


\section{RESUMEN GRÁFICO}

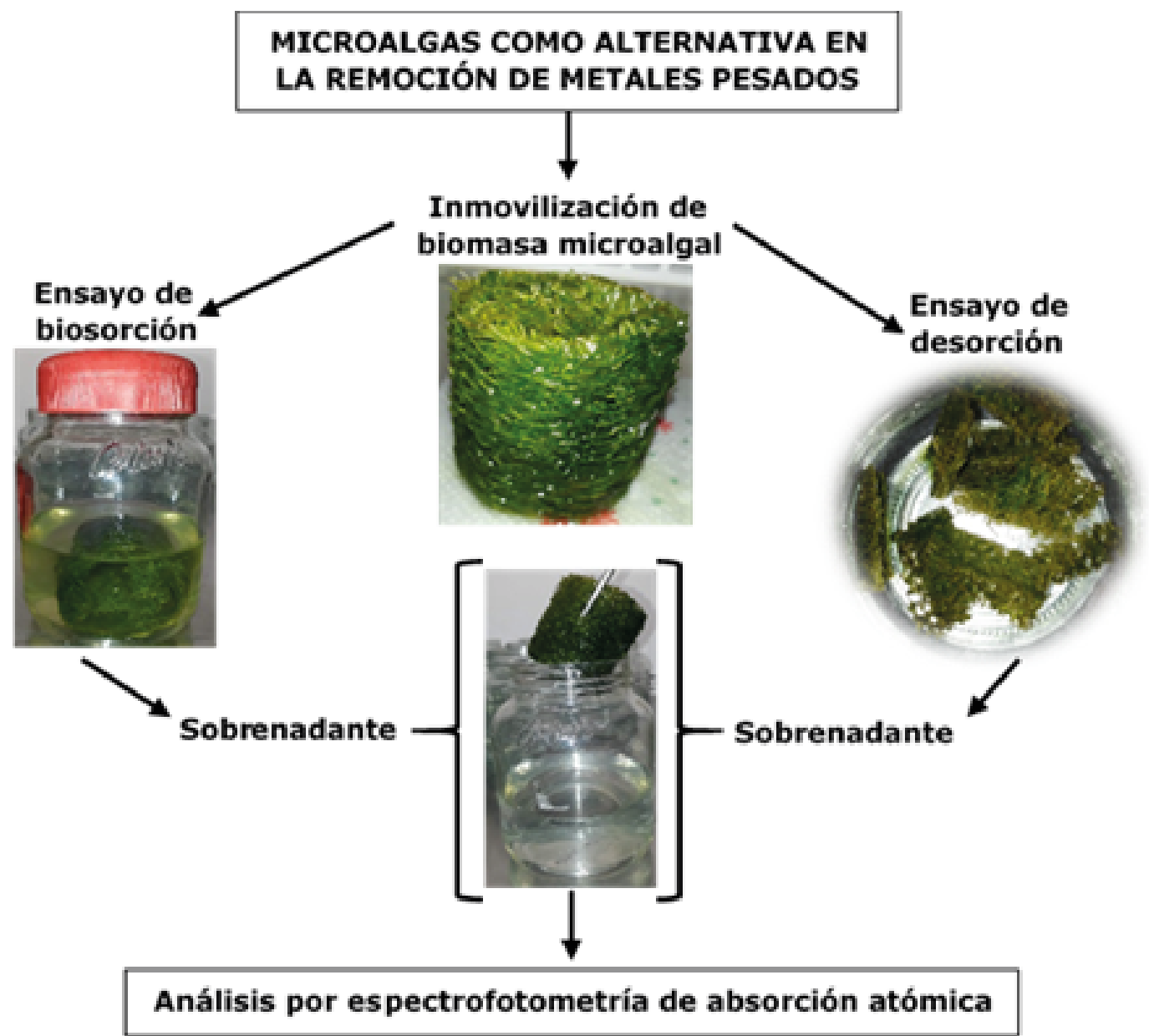

Fuente: Autores

\section{INTRODUCCIÓN}

La contaminación de todos los compartimentos ambientales con metales pesados, producto de las diversas actividades humanas, es un problema ambiental que se ha agudizado a nivel mundial (Reyes et al., 2016) y especialmente en Colombia, dado que cada día se generan aplicaciones excesivas de sustancias químicas para el aprovechamiento de minerales como el oro, y prácticas inadecuadas de disposición de aguas residuales industriales y agrícolas en cuerpos de aguas fundamentales para la dinámica poblacional de animales, vegetales y humanos (Vargas y Marrugo, 2019).

Todo lo anterior acompañado de la flexibilidad de las leyes nacionales en materia de protección del medio ambiente y la salud humana, ya que los niveles de contaminación máximos por metales pesados, permitidos en vertimientos de agua, que fueron establecidos por el Ministerio de Ambiente y Desarrollo Sostenible a través de la Resolución 0631 (2015) son: cadmio ( $\mathrm{Cd}=$ $0,10 \mathrm{mg} / \mathrm{L})$, mercurio $(\mathrm{Hg}=0,02 \mathrm{mg} / \mathrm{L})$ y plomo $(\mathrm{Pb}=0,50 \mathrm{mg} / \mathrm{L})$. Estos valores están muy por encima de los valores permitidos a nivel internacional. Por ejemplo, la Agencia de Protección Ambiental de los Estados Unidos [USEPA] estableció valores máximos de metales pesados en $\mathrm{Cd}$ de $0.01 \mathrm{mg} / \mathrm{L}$, $\mathrm{Hg}$ de $0,00003 \mathrm{mg} / \mathrm{L}$ y $\mathrm{Pb}$ de $0,006 \mathrm{mg} / \mathrm{L}$ (Nguyen et al., 2013), lo que manifiesta la negligencia del estado colombiano. Lo antes expuesto se corrobora con los trabajos desarrollados por Díaz-Arriaga (2014), quien halló en agua de consumo humano niveles de mercurio que superan los 0,002 mg/L y en aguas superficiales, niveles superiores al 
$0,003 \mathrm{mg} / \mathrm{L}$. Autores como Vargas y Marrugo (2019), encontraron en pescado niveles de mercurio superiores al $0,5 \mu \mathrm{g} / \mathrm{g}$ establecido por la OMS.

Sin embargo, existen muchas técnicas de remoción de metales pesados de las fuentes hídricas (Tahmasebi et al., 2015) de elevada eficiencia, pero que demandan un elevado costo económico y ambiental (Tang et al., 2018). Aun así, su uso es indispensable dada la alta toxicidad, rápida acumulación, nula degradabilidad (Awual et al., 2014; Staun et al., 2018) y gran movilidad de los metales pesados (Gutiérrez-Benítez et al., 2014), ya que pueden causar daño a tejidos, órganos, sistemas, alteraciones en el desarrollo, cáncer e incluso la muerte (Awual et al., 2016; Crowe et al., 2017). Por tal motivo se hace necesario y urgente la búsqueda de alternativas de biosorción de los contaminantes metálicos (Zhao et al., 2016) que sea de bajo costo y respetuosa con el ambiente, siendo la biosorción una alternativa que cumple con estos requerimientos, ya que su uso es simple, los materiales son asequibles y el bioadsorbente puede regenerarse (Renu et al., 2017).

La tecnología actual ha permitido el desarrollo de gran variedad de adsorbentes. Las investigaciones científicas actuales han volcado la mirada a los bioadsorbentes como las microalgas, debido a su gran variedad, abundancia, disponibilidad de las diferentes especies, buena capacidad para absorber iones metálicos (Yin et al., 2019; GutiérrezBenítez et al., 2014), alta eficiencia, bajo costo y respeto con el ambiente (Yin et al., 2019; Vitola et al., 2018). Además, cuentan con diferentes mecanismos bioquímicos de captación de metales pesados (Lee \& Fisher, 2017) y neutralización de la toxicidad (Yin et al., 2019; Bilal et al., 2018). No obstante, el crecimiento de las microalgas está limitado por factores como la carga contaminante, la presencia de nutrientes, la salinidad, el pH, la luz, $\mathrm{CO}_{2}$, etc.

Ya que son organismos fotoautótrofos, que se ha recomendado la inmovilización de la microalga para brindarle protección y minimizar la contaminación biológica (bacterias u hongos) al crear una barrera física (Kandilian et al., 2017; Covarrubias et al., 2012; De Bashan \& Bashan, 2010), y así aumentar la biosorción (Benítez et al., 2018; Hernández et al., 2018); especialmente si se emplean soportes naturales como el fruto seco de Luffa cylindrica, que tiene la ventaja de ser no tóxico, no reactivo (es decir, no interfiere con la actividad de la célula microalga), barato, mecánicamente fuerte, tiene alto grado de porosidad que favorece una mayor superficie de contacto, alto volumen específico y baja gravedad específica (propiedades físicas estables para cultivos a largo plazo) y es biodegradable (Nabizadeh et al., 2008; Liu et al., 1998).

La adopción de este tipo de enfoques de biorremediación es muy bien recibida, debido a su potencial de sostenibilidad (Emenike et al., 2017). Por consiguiente, el objetivo de esta investigación fue determinar la capacidad de biosorción y desorción de los metales pesados mercurio, cadmio y plomo por las microalgas Chlorella vulgaris y Scenedesmus obliquus, inmovilizadas en un soporte natural.

\section{MATERIALES Y MÉTODOS}

Inmovilización de la biomasa microalgal: Las microalgas utilizadas fueron Scenedesmus obliquus (SOLINM $98 \%$ ) y Chlorella vulgaris (CVLINM 99\%), aisladas desde el complejo cenagoso de Santiago Apóstol en el departamento de Sucre (Colombia), las cuales hacen parte de la colección del banco de germoplasma del Laboratorio de Investigaciones Microbiológicas de la Universidad de Sucre, inmovilizadas en fruto seco de Lufa cylindrica como soporte y previamente lavado con detergente para retirar impurezas (Nabizadeh et al., 2008). Posteriormente, se cortaron y esterilizaron fragmentos de microalgas con $30,0 \pm 1$ $\mathrm{mm}$ de diámetro y $35,0 \pm 1 \mathrm{~mm}$ de grosor, se impregnaron con un medio de cultivo nutrifoliar (Colinagro 4,0 ) que contiene 200 $\mathrm{g} / \mathrm{L}$ de nitrógeno total, $100 \mathrm{~g} / \mathrm{L}$ de fósforo y nutrientes como K, Mg, S, Cl, Fe, Cu, $\mathrm{Zn}, \mathrm{Mn}$, B y Mo por 24 h (Hernández et al., 2018) $y$, finalmente, se introdujeron los metales pesados en la solución con microalgas, en fase estacionaria (20 días), por 24 días. Después se retiraron los fragmentos de microalgas inmovilizadas, se lavaron para eliminar el exceso de material biológico y la biomasa inmovilizada se determinó por la diferencia 
del peso del estropajo antes y después de la inmovilización (Akhtar et al., 2004).

Capacidad de biosorción: se determinó inoculando a las microalgas inmovilizadas (en los soportes de estropajo) con las soluciones de metales pesados en forma de nitratos (grado analítico): $\mathrm{Cd}\left(\mathrm{NO}_{3}\right)_{2} \times 4 \mathrm{H}_{2} \mathrm{O}$, $\mathrm{Pb}\left(\mathrm{NO}_{3}\right)_{2}$ y $\mathrm{Hg}\left(\mathrm{NO}_{3}\right)_{2}$ a concentraciones de $4,0 \mathrm{mg} / \mathrm{L}, 5,0 \mathrm{mg} / \mathrm{L}$ y $6,0 \mathrm{mg} / \mathrm{L}$, por un tiempo de $24 \mathrm{~h}$ de biosorción con luz constante (2000 lux). Pasado este tiempo, se retiraron los fragmentos de estropajos con las microalgas inmovilizadas y se determinaron los porcentajes de biosorción mediante análisis de los sobrenadantes de cada tratamiento, usando técnicas espectroscópicas propias para cada metal pesado (Benítez et al., 2018; Hernández et al., 2018). Los datos obtenidos de la capacidad de biosorción se tabularon en Excel para su análisis estadístico e interpretación de los resultados.

Capacidad de desorción: los fragmentos de estropajo con las microalgas inmovilizadas, después de aplicar los ensayos de biosorción, se utilizaron para determinar la capacidad de desorción de los metales pesados. Estos fragmentos se mezclaron con 50 $\mathrm{mL}$ de una solución compuesta por 0,1 M de HNO3 y EDTA, mantenida en agitación a $400 \mathrm{rpm}$ durante $55 \mathrm{~min}$ a $55^{\circ} \mathrm{C}$, que posteriormente se filtró (Kumar et al., 2018). La concentración final de iones metálicos se determinó en la fase acuosa, filtrada usando un espectrofotómetro de absorción atómica. La relación de desorción se calculó a partir de la cantidad de metal pesado desorbido por el eluyente y la capacidad de biosorción (ecuación 1):

\section{Tasa de desorción (\%)= [HMd/HMa] x100}

En la que HMd es la cantidad de metal pesado desorbido y $\mathrm{HMa}$ es la capacidad de biosorción (Ahmad et al., 2018). Los datos obtenidos de la capacidad de desorción se tabularon en Excel para su análisis estadístico e interpretación de los resultados.

Análisis de los metales pesados: la determinación de los metales pesados se realizó con un espectrofotómetro de absorción atómica, de llama aire-acetileno, para el análisis de cadmio (Cd) y plomo (Pb), y con un espectrofotómetro de absorción atómica, con vapor frío, para mercurio $(\mathrm{Hg})$. Las concentraciones bajas fueron determinadas por la técnica de tubo de grafito y deuterio, todo de acuerdo al Standard Addition Method.

Análisis estadístico: Los resultados se expresaron como la media \pm Desviación Estándar, se realizó un análisis de varianza, mediante un diseño completamente al azar, con arreglo factorial $2 \times 3$. El primer factor fueron las especies de microalgas (Scenedesmus obliquus y Chlorella vulgaris) y el segundo, las concentraciones de los metales pesados ( 4,5 y $6 \mathrm{mg} / \mathrm{L})$; previamente determinando el criterio de normalidad mediante la prueba de Shapiro Wilk (5\%). Las diferencias estadísticas significativas se determinaron mediante la prueba de Tukey $(p<0,05)$. Todos los experimentos se realizaron por quintuplicado y se analizaron en el software InfoStat versión libre.

\section{RESULTADOS Y DISCUSIÓN}

Los resultados de la capacidad de biosorción de metales pesados presentaron diferencias significativas entre las especies de microalgas Scenedesmus obliquus y Chlorella vulgaris, mostrando esta última la mayor capacidad de biosorción de metales pesados (Figura 1 A y B) con la concentración de 6,0 $\mathrm{mg} / \mathrm{L}$ (aunque esta concentración no mostró diferencias significativas con la de $5,0 \mathrm{mg} / \mathrm{L}$ ) (Figura $1 \mathrm{~B}$ ). El metal pesado más removido fue el $\mathrm{Cd}$, seguido de $\mathrm{Pb}$ y finalmente $\mathrm{Hg}$ (Figura 1 A). Sin embargo, la biosorción de todos los metales pesados por las microalgas fue superior al $77 \%$.

Estos resultados son consistentes con lo reportado por otros estudios. Dinesh et al. (2013) encontraron un $25 \%$ más de biosorción de $\mathrm{Ni}$ en sistemas acuosos por la microalga $C$. sorokiniana cuando se inmovilizó en fibras de estropajo, en comparación con la microalga no inmovilizada. Kumar et al. (2018) hallaron que Chlorella vulgaris pudo remover alrededor del $97 \%$ del $\mathrm{Cd}$, cuando fue expuesta a una dosis de $10 \mathrm{mg} / \mathrm{L}$. Hernández et al., (2018) reportan que Chlorella sp removió 95,4\% de mercurio y $93,4 \%$ de níquel, mostrando los mayores porcentajes de biosorción cuando la microalga estaba inmovilizada en el fruto seco de Luffa cylindrica; al igual que Benítez 


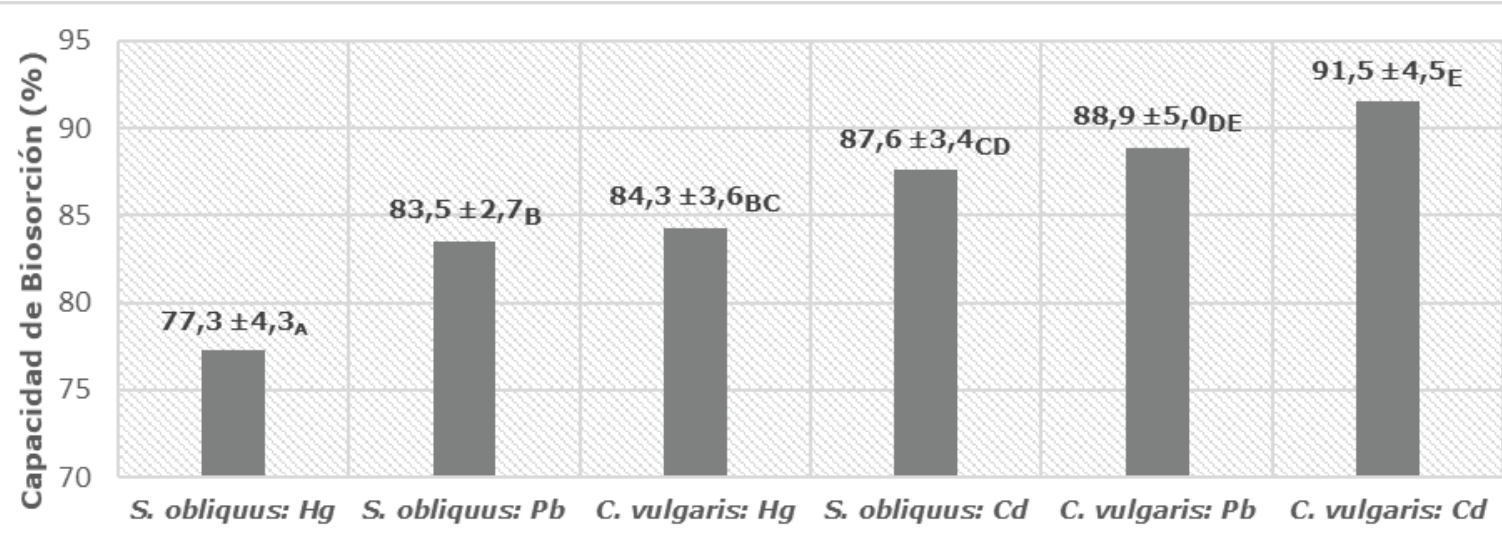

Especie microalgas* Metales pesados

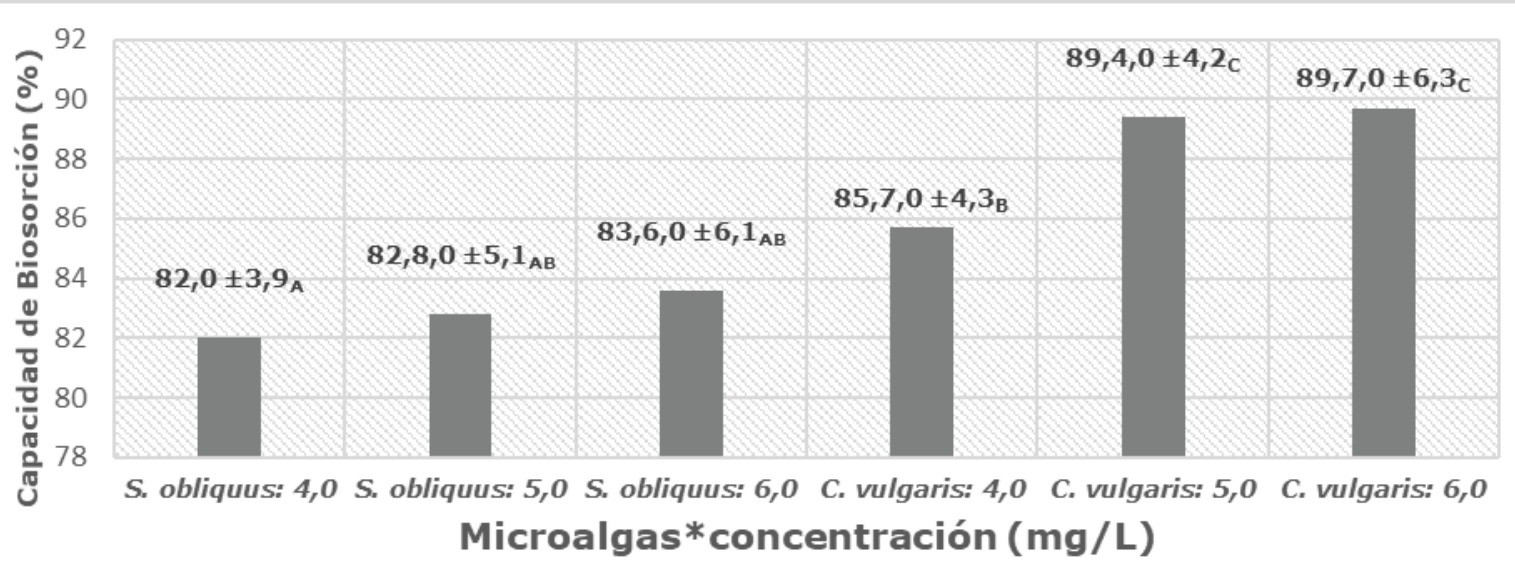

Figura 1. Resultados de la capacidad de biosorción de metales pesados por Chlorella vulgaris y Scenedesmus obliquus. A: biosorción entre las especies de microalgas y los metales pesados. B: entre las especies de microalgas y las concentraciones de los metales pesados.

\section{Fuente: Autores.}

et al., (2018), quienes sostienen que esta misma especie de microalga, inmovilizada en fragmentos de Luffa cylindrica, tuvo una biosorción del 98,9\% de mercurio. Dinesh et al. (2013) informan una biosorción del $98 \%$ de Zn cuando se empleó Chlorella marina inmovilizada en perlas de alginato. Adicionalmente, se ha encontrado que otras especies de microalgas, como Tetraselmis $s p$ inmovilizada, han removido hasta el $41 \%$ de nitrato y el $18 \%$ de silicato a las $3 \mathrm{~h}$ de exposición a aguas residuales de curtiduría (Adam et al., 2015) y Chlorella marina, inmovilizada en perlas de alginato, ha logrado la biosorción hasta del $90 \%$ de nitrato y el 60 $\%$ de fosfato a las $24 \mathrm{~h}$ de exposición (Dinesh et al., 2013).

Los resultados de la capacidad de desorción de los metales pesados, examinando la biomasa microalgal inmovilizada, muestran diferencias significativas entre las especies de microalgas. La tabla 1 muestra diferencias significativas entre los metales pesados, presentando Chlorella vulgaris la mayor capacidad de desorción de mercurio, con $97,29 \pm 1,93 \%$ del mercurio removido, seguido de cadmio con $96,86 \pm 2,14 \%$ y Plomo con 95,48 $\pm 1,19 \%$; mientras que Scenedesmus obliquus mostró una desorción de $96,74 \pm 2,14 \%$ de Mercurio, 95,15 $\pm 2,90 \%$ de cadmio y $93,82 \pm 2,68 \%$ de Plomo removido. Lo anterior indican que el mercurio fue el metal pesado con mayores porcentajes de desorción, seguido de cadmio y por último plomo. Estos resultados son consistentes a los reportado por Benítez et al. (2018), quienes reportan una desorción del $84.1 \%$ del Mercurio removido por Chlorella sp. Mientras que Kumar et al. (2018) hallaron una desorción de alrededor del $87 \%$ del Cd removido por la biomasa de Chlorella vulgaris. 
Tabla 1. Resultados de la capacidad de desorción de metales pesados desde la biomasa microalgas inmovilizada.

\begin{tabular}{crr} 
Metales pesados & \multicolumn{2}{c}{ Capacidad de Desorción (\%) } \\
\cline { 2 - 3 } $\mathrm{Hg}$ & $97,28 \pm 1,93 \mathrm{~b}$ & Scenedesmus obliquus \\
$\mathrm{Cd}$ & $96,86 \pm 2,14 \mathrm{~b}$ & $96,74 \pm 2,14 \mathrm{~b}$ \\
$\mathrm{~Pb}$ & $95,48 \pm 1,19 \mathrm{ab}$ & $95,15 \pm 2,90 \mathrm{ab}$ \\
& & $93,82 \pm 2,68 \mathrm{a}$
\end{tabular}

Valores seguidos de letras diferentes difieren en forma significativa $(P<0,05)$.

Fuente: Autores

\section{CONCLUSIONES}

El uso de microalgas inmovilizadas, como técnicas de biosorción de metales pesados, es eficiente, dado que las microalgas empleadas en esta investigación permitieron una biosorción superior al $74 \%$ de los metales pesados, y una desorción superior al $93 \%$ de los mismos. Chlorella vulgaris mostró los mayores promedios de absorción con 94,77 $\%$ de cadmio, $92.45 \%$ de plomo y $87.61 \%$ de mercurio, lo que indica que esta especie de microalga posee mayor afinidad hacia estos metales pesados, en comparación con Scenedesmus obliquus.

\section{CONTRIBUCIÓN DE LA AUTORÍA}

Deimer Vitola Romero: investigación, análisis de datos, escritura - borrador original. Alexander Pérez Cordero: investigación, conceptualización, escritura - revisión y edición. Donicer E. Montes Vergara: análisis de datos, revisión y edición.

\section{AGRADECIMIENTOS}

Los autores de este manuscrito extienden sus agradecimientos a la Universidad de Sucre, al Laboratorio de Investigaciones Microbiológicas y al grupo de investigación Bioprospección Agropecuaria, quienes fueron las entidades financiadoras.

\section{CONFLICTO DE INTERESES}

La información contenida en este manuscrito es original, revisada y preparada por las personas que participan en el mismo. Declaramos que no existe conflicto de intereses que ponga en riesgo la validez de los resultados del mismo.

\section{LITERATURA CITADA}

Adam, S., Suresh, P., Santhanam, P., Dinesh, S. \& Prabhavathi, P. (2015). Bioremediation of tannery wastewater using immobilized marine microalga Tetraselmis $s p$ : experimental studies and pseudo-second order kinetics. Journal of Marine Biology \& Oceanography, 4(1), 1-11. http://dx.doi. org/10.4172/2324-8661.1000141

Ahmad, A., Bhat, A. H. \& Buang, A. (2018). Biosorption of transition metals by freely suspended and $\mathrm{Ca}$-alginate immobilised with Chlorella vulgaris: Kinetic and equilibrium modeling. Journal of Cleaner Production, 171, 1361-1375. https://doi.org/10.1016/j. jclepro.2017.09.252

Akhtar, N., Iqbal, J. \& Iqbal, M. (2004). Removal and recovery of nickel(II) from aqueous solution by loofa spongeimmobilized biomass of Chlorella sorokiniana: characterization studies. Journal of Hazardous Materials, 108(1-2), 85-94. https://doi. org/10.1016/j.jhazmat.2004.01.002

Awual, R., Hasan, M., Khaleque, A. \& Sheikh, C. (2016). Treatment of copper(II) containing wastewater by a newly developed ligand based facial conjugate materials. Chemical Engineering Journal, 288, 368-376. https://doi.org/10.1016/j.cej.2015.11.108

Awual, R., Ismael, M., Khaleque, A. \& Yaita, T. (2014). Ultra-trace copper(II) detection and removal from wastewater using novel 
meso-adsorbent. Journal of Industrial and Engineering, 20(4), 2332-2340. https://doi. org/10.1016/j.jiec.2013.10.009

Benítez, S., Pérez, A. \& Vitola, D. (2018). Removal and recovery of mercury in vitro using immobilized live biomass of Chlorella sp. Indian Journal of Science and Technology, 11(45), 1-8. https://doi.org/10.17485/ ijst/2018/v11i45/137575

Bilal, M., Rasheed, T., Sosa-Hernández, J. E., Raza, A., Nabeel, F. \& Iqbal, H. M. N. (2018). Biosorption: an interplay between marine algae and potentially toxic elements-a review. Marine Drugs, 16(2), p. 65. https:// doi.org/10.3390/md16020065

Covarrubias, S. A., De Bashan, L. E., Moreno, M. \& Bashan, Y. (2012). Alginate beads provide a beneficial physical barrier against native microorganisms in wastewater treated with immobilized bacteria and microalgae. Applied Microbiology and Biotechnology, 93(6), 2669-2680. https:// doi.org/10.1007/s00253-011-3585-8

Crowe, W., Allsopp, P. J., Watson, G. E., Magee, P. J., Strain, J. J., Armstrong, D. J., Ball, E. \& McSorley, E. M. (2017). Mercury as an environmental stimulus in the development of autoimmunity- A systematic review. Autoimmunity Reviews, 16(1), 72-80. https://doi.org/10.1016/j. autrev.2016.09.020

De Bashan, L. E. \& Bashan, Y. (2010). Immobilized microalgae for removing pollutants: Review of practical aspects. Bioresource Technology, 101(6), 1611-1627. https://doi.org/10.1016/j. biortech.2009.09.043

Díaz-Arriaga, F. A. (2014). Mercurio en la minería del oro: impacto en las fuentes hídricas destinadas para consumo humano. Revista de Salud Pública, 16(6), 947-957. http:// dx.doi.org/10.15446/rsap.v16n6.45406

Dinesh, S., Santhanam, P., Jayalakshmi, T., Nandakumar, R., Ananth, S., Devi, A. S. \& Balaji , B. (2013). Optimization of pH and retention time on the removal of nutrients and heavy metal (zinc) using immobilized marine microalga Chlorella marina. Journal of Biological Sciences, 13(5), 400-405. https:// dx.doi.org/10.3923/jbs.2013.400.405
Emenike, C. U., Agamuthu, P. \& Fauziah, S. H. (2017). Sustainable remediation of heavy metal polluted soil: A biotechnical interaction with selected bacteria species. Journal of Geochemical Exploration, 182, 275-278. gexplo.2016.10.002

Gutiérrez-Benítez, O., González-Álvarez, J., Freire-Leira, M. S., Rodríguez-Rico, I. L. y Moreira-González, A. R. (2014). Potencialidades de un biosorbente algal para la remoción de metales pesados. Tecnología Química, 34(1), 82-93. https://www. redalyc. org/pdf/4455/445543781008.pdf

Hernández, Y., Pérez, A. \& Vitola, D. (2018). Biosorption of mercury and nickel in vitro by microalga Chlorella $s p$. in solution and immobilized in dry fruit of squash (Luffa cylindrica). Indian Journal of Science and Technology, 11(41), 1-8 https://dx.doi. org/10.17485/ijst/2018/v11i41/131111

Kandilian, R., Jesus, B., Legrand, J., Pilon, L. \& Pruvost, J. (2017). Light transfer in agar immobilized microalgae cell cultures. Journal of Quantitative Spectroscopy and Radiative Transfer, 198, 81-92. https://doi. org/10.1016/j.jqsrt.2017.04.027

Kumar, M., Singh, A. K. \& Sikandar, M. (2018). Study of sorption and desorption of Cd (II) from aqueous solution using isolated green algae Chlorella vulgaris. Applied Water Science, 8, 225. https://doi.org/10.1007/ s13201-018-0871-y

Lee, C. S. \& Fisher, N. S. (2017). Bioaccumulation of methylmercury in a marine diatom and the influence of dissolved organic matter. Marine Chemistry, 197, 70-79. https://doi.org/10.1016/j. marchem.2017.09.005

Liu, Y. K., Seki, M., Tanaka, H. \& Furusaki, S. (1998). Characteristics of loofa (Lufa cylindrica) sponge as a carrier for plant cell immobilization. Journal of Fermentation and Bioengineering, 85(4), 416-421. https://doi. org/10.1016/S0922-338X(98)80086-X

Nabizadeh, R., Naddafi, K., Mesdaghinia, A. \& Nafez, A. H. (2008). Feasibility study of organic matter and ammonium removal 
using loofa sponge as a supporting medium in an aerated submerged fixed-film reactor (ASFFR). Electronic Journal of Biotechnology, 11(4), 1-9. https://www.scielo.cl/pdf/ejb/ v11n4/a06.pdf

Nguyen, T. A. H., Ngo, H. H., Guo, W. S., Zhang, J., Liang, S., Yue, Q. Y., Li, Q. \& Nguyen, T. V. (2013). Applicability of agricultural waste and by-products for adsorptive removal of heavy metals from wastewater. Bioresource Technology, 148, 574-585. https://doi. org/10.1016/j.biortech.2013.08.124

Renu, K., Madhyastha, H., Madhyastha, R., Maruyama, M., Arunachlam, S. \& Abilash, V. G. (2018). Role of arsenic exposure in adipose tissue dysfunction and its possible implication in diabetes pathophysiology, Toxicology Letters, 284, 86-95. https://doi. org/10.1016/j.toxlet.2017.11.032

Resolución 0631 de 2015. (2015, 17 de marzo). Ministerio de Ambiente y Desarrollo Sostenible. Diario Oficial No. 49.486. http:// www.emserchia.gov.co/PDF/Resolucion631. pdf

Reyes, Y. C, Vergara, I., Torres, O. E., Díaz, M. y González, E. E. (2016). Contaminación por metales pesados: implicaciones en salud, ambiente y seguridad alimentaria. Revista Ingeniería, Investigación y Desarrollo, 16(2), 66-77. https://revistas.uptc.edu. co/index.php/ingenieria_sogamoso/article/ view/5447/4518

Staun, C., Vaughan, J., López-Anton, M. A., Rumayor, M. \& Martínez-Tarazona, M. R. (2018). Geochemical speciation of mercury in bauxite. Applied Geochemistry, 93, 30-35. https://doi.org/10.1016/j. apgeochem.2018.03.007
Tahmasebi, E., Masoomi, M. Y., Yamimi, Y. \& Morsali, A. (2015). Application of mechanosynthesized azine-decorated zinc (II) metal-organic frameworks for highly efficient removal and extraction of some heavy-metal ions from aqueous samples: a comparative study. Inorganic Chemistry, 54(2), 425-433. https://doi.org/10.1021/ ic5015384

Tang, J., He, J., Xin, X., Hu, H. \& Liu, T. (2018). Biosurfactants enhanced heavy metals removal from sludge in the electrokinetic treatment. Chemical Engineering Journal, 334, 2579-2592. https://doi.org/10.1016/j. cej.2017.12.010

Vargas, S. P. y Marrugo, J. L. (2019). Mercurio, metilmercurio y otros metales pesados en peces de Colombia: riesgo por ingesta. Acta Biológica Colombiana. 24(2), 232-242. http://dx.doi.org/10.15446/abc. v24n 2.74128

Vitola, D., Pérez, A.. \& Oviedo, Y. (2018). Biodegradation activity of crude oil by Chlorella sp. under mixotrophic conditions. Indian Journal of Science and Technology, 11(29), 1-8. https://doi.org/10.17485/ ijst/2018/v11i29/127832

Yin, K., Wang, Q., Lv, M. \& Chen, L. (2019). Microorganism remediation strategies towards heavy metals. Chemical Engineering Journal, 360, 1553-1563. https://doi.org/10.1016/j. cej.2018.10.226

Zhao, J., Liu, J., Li, N., Wang, W., Nan, J., Zhao, Z. \& Cui, F. (2016). Highly efficient removal of bivalent heavy metals from aqueous systems by magnetic porous Fe304$\mathrm{MnO} 2$ : Adsorption behavior and process study. Chemical Engineering Journal, 304, 737-746. https://doi.org/10.1016/j.cej.2016.07.003
Conflicto de intereses

Los autores declaran no tener ningún conflicto de intereses.

\section{(9) $(1)(\Theta$}


